\title{
Clinical characteristics and prognostic value of the KRAS G12C mutation in Chinese non- small cell lung cancer patients
}

\author{
Si-Yang Liu', Hao Sun', Jia-Ying Zhou', Guang-Ling Jie', Zhi Xie', Yang Shao², Xian Zhang², Jun-Yi Ye²,
} Chun-Xiang Chen ${ }^{3}$, Xu-Chao Zhang ${ }^{1}$, Qing Zhou', Jin-Ji Yang ${ }^{1}$ and Yi-Long Wu ${ }^{1 *}$ (D

\begin{abstract}
Background: The KRAS mutation is the second most common genetic variant in Chinese non-small cell lung cancer (NSCLC) patients. At the 2019th World Conference of Lung Cancer, the KRAS G12C-specific inhibitor AMG510 showed promising results in the phase I clinical trial. However, the frequency, clinical characteristics, and prognostic significance of the KRAS G12C mutation in Chinese NSCLC patients are rarely reported.

Methods: Next-generation sequencing was used to confirm the KRAS mutation status in 40,804 NSCLC patients from multiple centers (mCohort). Survival data were collected retrospectively from 1456 patients at one of the centers, the Guangdong Lung Cancer Institute (iCohort).
\end{abstract}

Results: In the mCohort, 3998 patients (9.8\%) were confirmed to harbor a KRAS mutation, of whom 1179 (29.5\%) had the G12C subtype. In the iCohort, 130 NSCLC patients (8.9\%) had a KRAS mutation and 42 (32.3\%) had the G12C subtype. The G12C subgroup included more male patients $(85.2 \%$ vs $67.4 \%, P<0.0001)$ and more smokers $(76.2 \%$ vs $53.4 \%, P=0.02)$ than did the non-G12C subgroup. Both the KRAS mutation group and KRAS G12C mutation subgroup were associated with a shorter median overall survival (OS) than wildtype tumors (15.1 vs 26.7 months, hazard ratio $[H R]_{K R A S}=1.50, P=0.002 ; 18.3$ vs 26.7 months, $\left.H R_{G 12 C}=1.66, P=0.007\right)$. In Cox regression analysis, smoking $(H R=1.39, P=0.05)$ and stage IV disease $(H R=2.72, P<0.001)$ remained as independent predictors of shorter OS. Both the KRAS mutation ( $H R=1.30, P=0.07)$ and KRAS G12C mutation $(H R=1.47, P=0.07)$ reached borderline significance.

Conclusions: In the largest sample used thus for, our study found that approximately $10 \%$ of Chinese NSCLC patients had KRAS mutations. Of these, nearly 30\% harbored the KRAS G12C mutation subtype, which was most common in male smokers. The KRAS G12C mutation is a biomarker of poor prognosis in Chinese NSCLC patients, which could potentially be improved by G12C-specific inhibitors in the future.

(296 words)

Keywords: KRAS mutation, KRAS G12C mutation, Prognosis, Non-small cell lung cancer, Chinese patients

\footnotetext{
* Correspondence: syylwu@live.cn

'Guangdong Lung Cancer Institute, Guangdong Provincial People's Hospital, Guangdong Academy of Medical Sciences, School of Medicine, South China University of Technology, 106 Zhongshan Er Road, Guangzhou 510080, China Full list of author information is available at the end of the article
}

(c) The Author(s). 2020 Open Access This article is licensed under a Creative Commons Attribution 4.0 International License, which permits use, sharing, adaptation, distribution and reproduction in any medium or format, as long as you give appropriate credit to the original author(s) and the source, provide a link to the Creative Commons licence, and indicate if changes were made. The images or other third party material in this article are included in the article's Creative Commons licence, unless indicated otherwise in a credit line to the material. If material is not included in the article's Creative Commons licence and your intended use is not permitted by statutory regulation or exceeds the permitted use, you will need to obtain permission directly from the copyright holder. To view a copy of this licence, visit http://creativecommons.org/licenses/by/4.0/ The Creative Commons Public Domain Dedication waiver (http://creativecommons.org/publicdomain/zero/1.0/) applies to the data made available in this article, unless otherwise stated in a credit line to the data. 


\section{Background}

Rapid developments have been achieved in the area of epidermal growth factor-receptor tyrosine kinase inhibitors (EGFR-TKIs) and immunotherapy with checkpoint inhibitors for lung cancer patients [1-5]. Although treatment resistance is inevitable, advances of third-generation EGFR-TKIs prolong the survival of patients with EGFR mutations. The KRAS mutation is the second most common genetic variant in Chinese non-small cell lung cancer (NSCLC) patients [6]. Many retrospective and prospective studies have attempted to treat KRAS mutation patients with EGFR-TKIs [7] and MAP-ERK kinase (MEK) inhibitors $[8,9]$, but none were successful. No targeted therapy is available for patients with KRAS mutations and chemotherapy remains the standard. Patients with KRAS mutations seemed to respond to checkpoint blockade therapy in several recently published studies [10-12]. In addition to immunotherapy with checkpoint inhibitors, KRAS $G 12 C$-specific inhibitors show promising preclinical and clinical results [13]. The World Conference of Lung Cancer in 2019 presented promising and up-to-date clinical data on the drug AMG510, which was given to 13 patients with non-small cell lung cancer (NSCLC) at a dose of 960 mg once per day. Seven patients achieved partial response and six achieved stable disease. The objective response rate was $54 \%$ and the disease control rate was $100 \%$. Additionally, a series of clinical trials targeting KRAS G12C mutations with $G 12 C$-specific inhibitors, including RMC4630 and MRTX849 are ongoing $[14,15]$.

It was reported that $30 \%$ of Caucasian NSCLC patients harbored KRAS mutations, of which $35 \sim 45 \%$ were of the $G 12 C$ subtype $[16,17]$. Therefore, the incidence of KRAS G12C mutations in NSCLC in those Caucasian patients was nearly $12 \%$. In Asians, however, the frequency of $K R A S$ G12C mutations has rarely been studied and the prognosis of those carrying the G12C mutation is still unclear. Here, we examined the incidence of this mutation subtype, and its clinical characteristics, in Chinese NSCLC patients drawn from two cohorts and explored the prognostic role of the KRAS G12C mutation.

\section{Methods}

\section{Patients}

From January 2016 to September 2019, the NGS results from 40,804 NSCLC patients from multiple centers (mCohort) were analyzed; of these patients, $3998 \mathrm{had}$ KRAS mutations. In total, 1776 patients with KRAS mutations had NGS results analyzed from tumor tissue, 1646 from tumor tissue and liquid biopsy, and 576 from liquid biopsy alone (e.g., peripheral blood, pleural effusion and cerebrospinal fluid). Clinical data of these patients were pooled retrospectively and the factors included in the analysis were age, sex, pathology, and clinical stage at the time of diagnosis. Smoking history and survival data of 1456 NSCLC patients from one of the centers, the Guangdong Lung Cancer Institute (iCohort), were collected retrospectively from the electronic medical records.

\section{Analysis}

The chi-square test was used to compare categorical data. Overall survival (OS) was measured from the date of pathological diagnosis of lung cancer to the date of death or last follow-up, with a cut-off date of September 2019. Kaplan-Meier survival curves were generated to estimate OS in different genomic groups. The univariate and multivariate Cox proportional hazards model was used to evaluate the prognostic value of KRAS and KRAS G12C mutations on OS. Statistical significance was defined as a $p$-value less than 0.05 .

\section{Results}

Frequency of KRAS G12C mutations in Chinese NSCLC patients

In the mCohort, 3998 NSCLC patients had KRAS mutations; 25 patients had two KRAS mutation subtypes, and the frequency of KRAS mutations was $9.8 \%$. Of the patients with KRAS mutations, 1179 (29.5\%) were confirmed to harbor G12C mutations (Fig. 1a). The proportions of the other three major KRAS codon 12 subtypes were as follows: $G 12 \mathrm{~V}, 18.3 \%(N=731)$; $G 12 D$, $17.3 \%(N=693)$; and $G 12 A, 8.4 \%(N=334)$ (Fig. 2).

In the iCohort, 130 of 1456 NSCLC patients (8.9\%) were confirmed to have KRAS mutations, of whom 42 (32.3\%) harbored G12C mutations; there were 304 wildtype patients (excluding EGFR mutation, $A L K$ fusion, ROS1 fusion and BRAF mutation) (Fig. 1b). The distribution of $K R A S$ mutation subtypes was comparable to that in the mCohort. For the other three major codon 12 subtypes, the proportions were as follows: G12D, $19.2 \%$ $(N=25) ; G 12 V, 13.1 \%(N=17)$; and $G 12 A, 6.9 \%(N=9)$ (Fig. 2).

\section{Clinical characteristics of patients with the KRAS G12C mutation}

The clinical and pathological characteristics of NSCLC patients with KRAS G12C and non-G12C KRAS mutations were compared in the mCohort (Table 1). The mean ages of the G12C and non-G12C subtype patients were 63 and 62 years old, respectively $(P=0.02)$. The proportion of male patients was higher in the $G 12 C$ subgroup than that in the non-G12C subgroup $(85.2 \%$ vs $67.4 \%, P<0.0001)$. Most of the G12C and non-G12C subtype patients were diagnosed with adenocarcinoma (rates were more than $90 \%$ in both groups). Nearly $40 \%$ of the patients were diagnosed with stage IV disease in the G12C and non- 


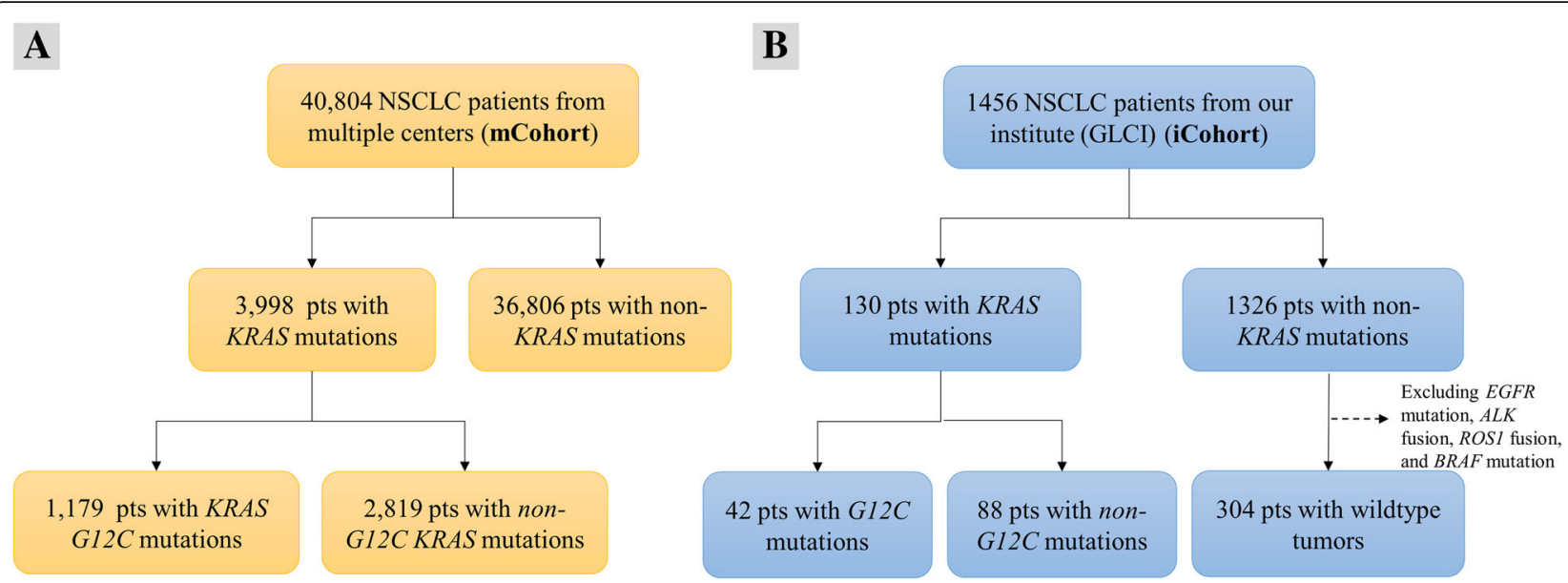

Fig. 1 Flow charts of NSCLC patient enrollment in the study. Patients included from multiple centers cohort (mCohort) a. Patients included and excluded from Guangdong Lung Cancer Institute cohort (iCohort) b. pts.: patients

G12C subgroups. In the iCohort, the clinical and pathological characteristics of the patients with $G 12 C$ and non-G12C mutations were comparable to those in the mCohort. Of note, in the iCohort, $76.2 \%$ of the patients in the $G 12 C$ subgroup were former or current smokers, compared with $53.4 \%$ of those in the non-G12C subgroup $(P=0.02)$.

The clinical characteristics of KRAS mutation and wildtype tumors were also compared in the iCohort. In the KRAS mutation and wildtype subgroups, 79.2 and $71.7 \%$ of the patients were male $(P=0.10)$, respectively, with median ages of 63 and 61 years $(P=0.01) ; 60.8$ and $53.3 \%$ were former or current smokers $(P=0.15)$, respectively. Most patients in both the KRAS mutation and wildtype subgroups had adenocarcinoma and stage IV disease.

\section{Prognostic value of the KRAS G12C mutation}

Survival data were collected retrospectively for 130 KRAS mutation and 304 wildtype patients from the
iCohort. In the Kaplan-Meier analysis, regardless of the KRAS mutation group or the KRAS G12C mutation subgroup, both were associated with a shorter median OS compared with wildtype tumors (15.1 vs 26.7 months, Hazard Ratio $[\mathrm{HR}]_{\text {KRAS }}=1.50$, $P=0.002 ; \quad 18.3$ vs 26.7 months, $\mathrm{HR}_{G 12 C}=1.66, \quad P=$ 0.007) (Fig. 3a, b).

To identify the prognostic values of the KRAS and KRAS G12C mutations on OS, clinical and molecular variables were included in Cox regression analysis. In the univariate analysis, age, male, smoker, stage IV disease, KRAS mutation, and KRAS G12C and non-G12C mutations were identified as independent factors for shorter OS (Table 2). In the multivariate Cox model, smoker $(\mathrm{HR}=1.39, P=0.05)$ and stage IV disease $(\mathrm{HR}=$ 2.72, $P<0.0001)$ remained as independent factors for poor prognosis. Both the KRAS mutation $(\mathrm{HR}=1.47$, $P=0.07)$ and the KRAS G12C mutation $(\mathrm{HR}=1.23, P=$ 0.07 ) were borderline statistically significant.

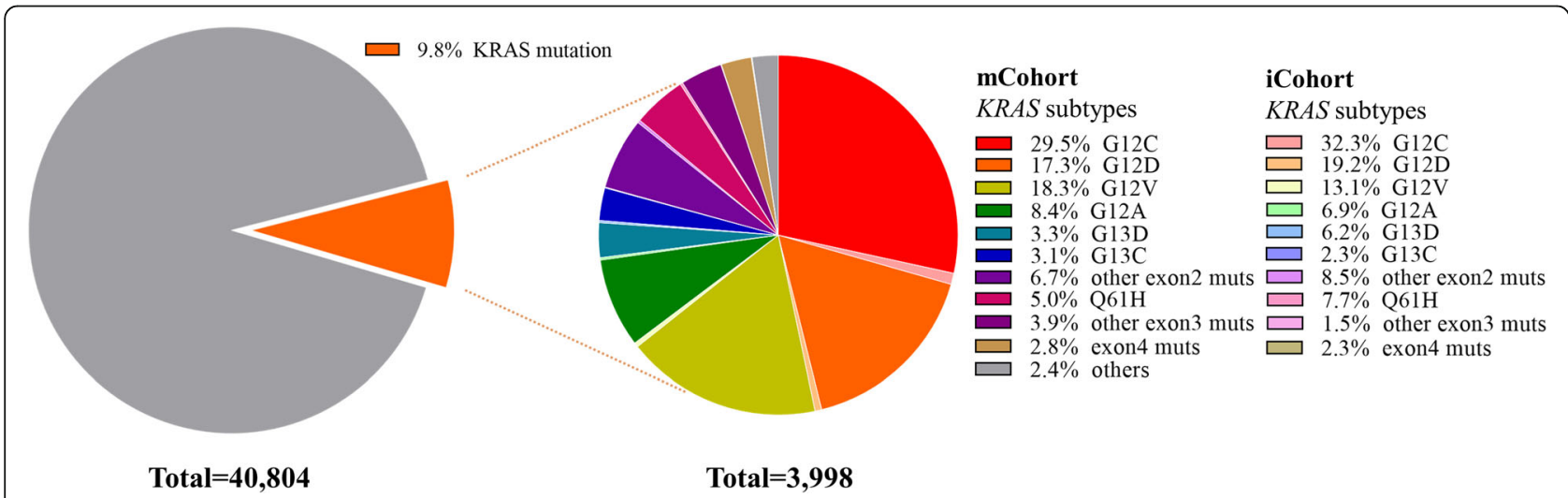

Fig. 2 Pie charts of NSCLC patients with KRAS mutations. Pie charts showing the proportions of KRAS mutation and wildtype tumors in the $\mathrm{mCohort} \mathrm{(left),} \mathrm{and} \mathrm{the} \mathrm{proportions} \mathrm{of} \mathrm{different} \mathrm{KRAS} \mathrm{mutation} \mathrm{subtypes} \mathrm{in} \mathrm{the} \mathrm{mCohort} \mathrm{(right)} \mathrm{which} \mathrm{included} \mathrm{patients} \mathrm{from} \mathrm{the} \mathrm{iCohort}$ 
Table 1 Clinical and pathological characteristics of KRAS G12C and non-G12C mutations from the mCohort

\begin{tabular}{llll}
\hline & KRAS G12C (N=1179) & Non-G12C (N=2819) & P-value \\
\hline $\begin{array}{l}\text { Age, mean (range) } \\
\text { Sex, } n \text { (\%) }\end{array}$ & $63(31-91)$ & $180)$ \\
Male & $1005(85.2 \%)$ & 8.02 \\
Female & $162(13.7 \%)$ & $875(31.0 \%)$ \\
NA & $12(1.0 \%)$ & $45(1.6 \%)$ \\
Pathology, $n$ (\%) & & \\
Adenocarcinoma & $1107(93.9 \%)$ & $2584(91.7 \%)$ \\
Squamous carcinoma & $23(2.0 \%)$ & $154(5.5 \%)$ \\
Adeno-squamous carcinoma & $9(0.8 \%)$ & $18(0.6 \%)$ \\
LCLC & $3(0.3 \%)$ & $13(0.5 \%)$ \\
Others & $37(3.1 \%)$ & $50(1.8 \%)$ \\
Stage, $n$ (\%) & & $85(3.0 \%)$ \\
I & $37(3.1 \%)$ & $86(3.1 \%)$ \\
II & $38(3.2 \%)$ & $235(8.3 \%)$ \\
III & $125(10.6 \%)$ & $1082(38.4 \%)$ \\
IV & $447(37.9 \%)$ & $1331(47.2 \%)$ \\
NA & $532(45.1 \%)$ & 0.24 \\
\hline
\end{tabular}

\# could not be computed, mCohort lung cancer patients from multiple centers, iCohort lung cancer patients from Guangdong Lung Cancer Institute, NA not available, LCLC large-cell lung cancer

\section{Discussion}

The frequency of KRAS mutations is much higher in Caucasian NSCLC patients, at around 30\%, than in Asian patients [18]. In our study, $9.8 \%$ of the patients in the mCohort harbored KRAS mutations, similar to the rates reported by Zhou's group [19]. EGFR and KRAS mutations are mutually exclusive, and Asian patients with NSCLC tend to have more EGFR mutations and thus fewer KRAS mutations [20]. The frequency of KRAS G12C mutations in Caucasians ranges from 35 to $45 \%$

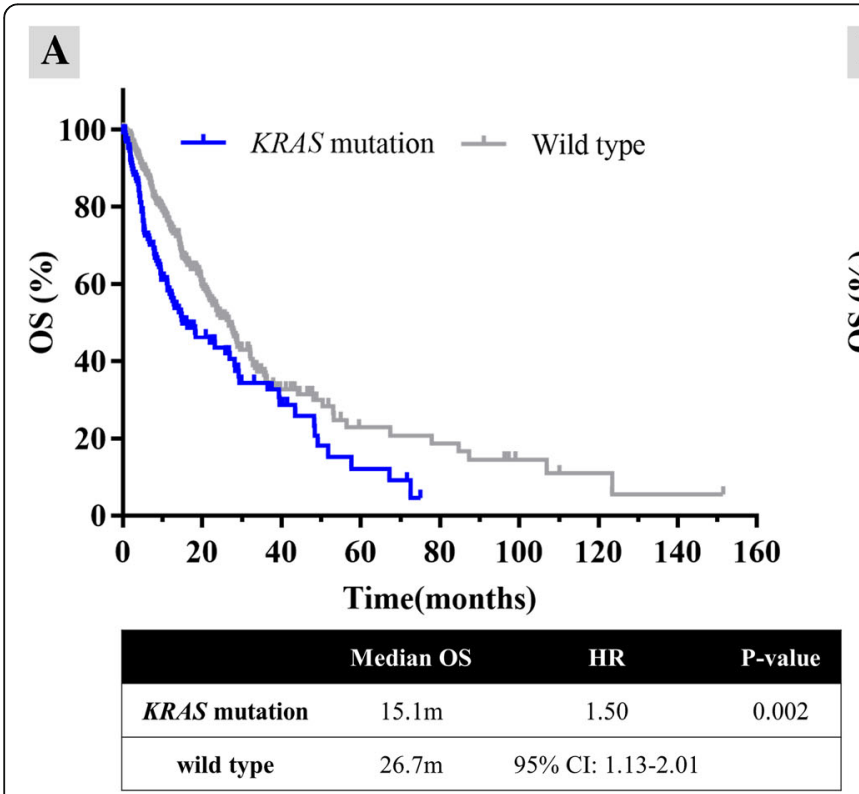

B

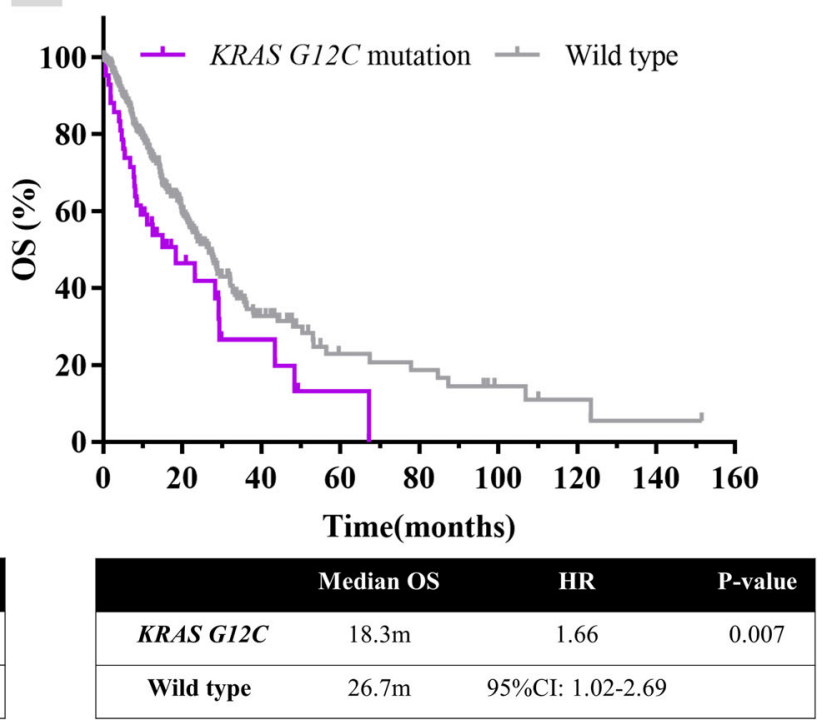

Fig. 3 Survival analysis of NSCLC patients with KRAS and KRAS G12C mutations. Overall survival (OS) analysis of KRAS mutation and wildtype tumors a. OS analysis of KRAS G12C mutation and wildtype tumors b. m: months; wt: wild type; HR: hazard ratio; Cl: confidence interval 
Table 2 Univariate and multivariate analysis of overall survival based on clinical and molecular variables

\begin{tabular}{|c|c|c|c|c|c|c|}
\hline \multirow[t]{2}{*}{ Variable } & \multicolumn{3}{|c|}{ Univariate analysis } & \multicolumn{3}{|c|}{ Multivariate analysis } \\
\hline & Crude HR & $95 \% \mathrm{Cl}$ & $P$ & Adjusted HR & $95 \% \mathrm{Cl}$ & $P$ \\
\hline Age & 1.02 & $1.00-1.03$ & 0.02 & 1.01 & $1.00-1.03$ & 0.07 \\
\hline \multicolumn{7}{|l|}{ Sex } \\
\hline Female & 1 & & & 1 & & \\
\hline Male & 1.41 & $1.05-1.90$ & 0.02 & 1.08 & $0.74-1.58$ & 0.71 \\
\hline \multicolumn{7}{|l|}{ Ever Smoking } \\
\hline Non-smoker & 1 & & & 1 & & \\
\hline Smoker & 1.46 & $1.13-1.90$ & 0.004 & 1.39 & $1.00-1.94$ & 0.05 \\
\hline \multicolumn{7}{|l|}{ Pathology } \\
\hline Adenocarcinoma & 1 & & & & & \\
\hline Squamous carcinoma & 1.16 & $0.71-1.88$ & 0.55 & & & \\
\hline others & 1.14 & $0.64-2.06$ & 0.65 & & & \\
\hline \multicolumn{7}{|l|}{ Stage } \\
\hline$|-| \mid$ & 1 & & & 1 & & \\
\hline III & 1.55 & $0.88-2.74$ & 0.13 & 1.58 & $0.89-2.81$ & 0.12 \\
\hline IV & 2.68 & $1.60-4.47$ & $* * *$ & 2.72 & $1.62-4.56$ & $* * *$ \\
\hline \multicolumn{7}{|l|}{ KRAS mutation } \\
\hline Wildtype & 1 & & & 1 & & \\
\hline Mutation & 1.51 & $1.16-1.98$ & 0.003 & 1.30 & $0.98-1.72$ & 0.07 \\
\hline \multicolumn{7}{|l|}{ KRAS mutation subtype } \\
\hline Wild-type & 1 & & & 1 & & \\
\hline G12C mutation & 1.65 & $1.10-2.47$ & 0.02 & 1.47 & $0.97-2.23$ & 0.07 \\
\hline Non-G12C mutation & 1.45 & $1.06-1.98$ & 0.02 & 1.23 & $0.90-1.69$ & 0.20 \\
\hline
\end{tabular}

NSCLC non-small cell lung cancer, HR hazard ratio

$[16,18,21,22]$. In our study, $29.5 \%$ of KRAS mutations in the mCohort were of the G12C subtype, which means that nearly $3 \%$ of these Chinese NSCLC patients harbored KRAS G12C mutations.

In Caucasians, KRAS mutations are more common in females and smokers [21-23]. Moreover, Dogan et al. and Osta et al. reported that $\mathrm{G} 12 \mathrm{C}$ mutations were more common in women and those with a smoking history $[17,24]$. By contrast, we found that male smokers more commonly harbored KRAS mutations, including G12C mutations, which is consistent with Guan et al. [25]. Although only a small proportion of KRAS mutation patients enrolled in the mCohort were diagnosed with stage I or II disease, KRAS mutation seems to be an early event that might drive lung cancer development $[18,21$, 22, 25]. Furthermore, the KRAS G12C mutation might be a drug target in early stage lung cancer.

The prognostic role of KRAS mutations in NSCLC patients in early and advanced stages is becoming clear. Two studies enrolled surgically resected lung adenocarcinoma patients and found that those with KRAS mutation tumors had worse disease free survival and OS compared with wildtype patients [21, 22]. Even after excluding EGFR mutations, a significant survival difference persisted. A poor prognosis of KRAS mutation patients in advanced lung cancer stages has also been reported [17, 26]. Guan et al., from our institute, enrolled stage I to IV lung cancer patients. To eliminate bias of disease stage, patients were randomly paired, and KRAS mutations still predicted a poor prognosis [25]. Our results are consistent with the available data indicating a shorter OS for KRAS mutations, compared to wildtype tumors. However, the prognostic role of KRAS G12C mutations has been rarely reported. Nadal et al. showed that the KRAS G12C mutation was associated with poor outcomes in surgically resected lung adenocarcinoma and remained an independent prognostic marker for OS in multivariate analysis [22]. Svaton et al. indicated that patients with $G 12 C$ mutations had shorter median OS compared to non-G12C KRAS mutation and wildtype patients (6.4 vs 10.3 vs 16.1 months, $P=0.01$ ) [26]. In our study, more than $80 \%$ of the NSCLC patients exhibited advanced disease. The median OS of the G12C mutation and wildtype patients was 18.3 and 26.7 months, respectively $(\mathrm{HR}=1.66, P=0.007)$. The prognostic value of the $G 12 C$ mutation was identified in Cox 
regression analysis. Although the $P$-value reached statistical margin, this may have been due to the small sample size of KRAS mutation patients in the iCohort. Patients with KRAS mutations could receive chemotherapy as standard treatment; some could choose immunotherapy with anti-PD-1/PD-L1 blockade. No other choices were available for clinicians to prescribe for these patients. Thus, detailed information regarding the treatment is not presented in Table 2. In general, the KRAS G12C mutation was a prognostic biomarker for poor OS in Chinese NSCLC patients.

Our study included the largest sample size of NSCLC patients harboring KRAS mutations thus far. However, it had a few limitations. First, we only had clinical and pathological data for the NSCLC patients from multiple centers and lacked survival data. Thus, the prognostic role of the KRAS G12C mutation in poor OS were taken from only one of the centers, namely, the iCohort. Second, in the iCohort, there were more stage IV patients in the KRAS mutation subgroup than in the wildtype subgroup, which may have affected the results where KRAS mutations were associated with a poor prognosis $(P=0.01)$. However, this result has been repeated in previously published studies. Similarly, although more patients had stage IV disease in the G12C subgroup than in the wildtype subgroup, the difference in their distribution did not reach statistical significance $(P=0.10)$. Thus, results regarding the prognostic roles of KRAS mutations and $G 12 C$ mutation were reliable. Third, racial differences in the KRAS G12C mutation should be explored further in future studies.

\section{Conclusion}

In general, our study identified that approximately $9 \%$ of Chinese NSCLC patients had KRAS mutations. Of these, nearly $30 \%$ harbored the KRAS G12C mutation subtype, which often occurred in male smokers. The KRAS G12C mutation predicted a poor OS, which could potentially be improved by specific $G 12 C$ inhibitors in the future.

\section{Abbreviations \\ Cl: Confidence interval; EGFR-TKIs: Epidermal growth factor-receptor tyrosine kinase inhibitors; HR: Hazard ratio; LCLC: Large cell lung cancer; MEK: MAP- ERK kinase; NGS: Next-generation sequencing; NSCLC: Non-small cell lung cancer; OS: Overall survival}

\section{Acknowledgements}

We thank Mr. Yang Shao, Mr. Xian Zhang, Mr. Jun-Yi Ye and Miss. ChunXiang Chen for providing data regarding patients with KRAS mutations from multiple centers.

\section{Authors' contributions}

Conception and design of the study: Prof. Yi-Long Wu; Acquisition of clinical data: Dr. Si-Yang Liu, Dr. Jia-Ying Zhou, Dr. Guang-Ling Jie and Dr. Hao Sun; Analysis and interpretation of the data: Dr. Si-Yang Liu, Mr. Yang Shao, Mr. Xian Zhang, Mr. Jun-Yi Ye and Miss. Chun-Xiang Chen; Manuscript drafting and revision: Dr. Si-Yang Liu and Prof. Yi-Long Wu; Final approval of the manuscript: All authors.

\section{Funding}

This work was supported by the National Key R\&D Program of China (2016YFC1303800 to Q. Zhou), Special Fund of Public Interest by National Health and Family Control Committee (201402031 to Y.L. Wu), Key Lab System Project of Guangdong Science and Technology Department and Guangdong Provincial Key Lab of Translational Medicine in Lung Cancer (2012A061400006, 2017B030314120 to Y.L. WU) and Health Collaborative Innovation Major Project from Guangzhou Science and Technology Bureau (201400000001-2 to Y.L. WU).

\section{Availability of data and materials}

The datasets used and analyzed during the current study are available from the corresponding author on reasonable request.

\section{Ethics approval and consent to participate}

The procedures in this study were approved by the Research Ethics Committee of Guangdong Provincial People's Hospital (2013185H). All patients provided written informed consent for the use of their NGS results.

\section{Consent for publication}

Not applicable.

\section{Competing interests}

The authors declare that they have no competing interests.

\section{Author details}

${ }^{1}$ Guangdong Lung Cancer Institute, Guangdong Provincial People's Hospital, Guangdong Academy of Medical Sciences, School of Medicine, South China University of Technology, 106 Zhongshan Er Road, Guangzhou 510080, China. ${ }^{2}$ Nanjing Geneseeq Technology Inc., Nanjing 210032, China. ${ }^{3}$ Burning Rock Biotech, Guangzhou 510000, China.

Received: 21 April 2020 Accepted: 29 May 2020

Published online: 25 June 2020

\section{References}

1. Ramalingam SS, Vansteenkiste J, Planchard D, Cho BC, Gray JE, Ohe Y, et al. Overall survival with Osimertinib in untreated, EGFR-mutated advanced NSCLC. N Engl J Med. 2020;382:41-50.

2. Gandhi L, Rodriguez-Abreu D, Gadgeel S, Esteban E, Felip E, De Angelis F, et al. Pembrolizumab plus chemotherapy in metastatic non-small-cell lung Cancer. N Engl J Med. 2018;378:2078-92.

3. Zhang C, Leighl NB, Wu YL, Zhong WZ. Emerging therapies for non-small cell lung cancer. J Hematol Oncol. 2019:12:45.

4. Qiu Z, Chen Z, Zhang C, Zhong W. Achievements and futures of immune checkpoint inhibitors in non-small cell lung cancer. Exp Hematol Oncol. 2019;8:19.

5. Akinleye A, Rasool Z. Immune checkpoint inhibitors of PD-L1 as cancer therapeutics. J Hematol Oncol. 2019;12:92

6. Gou LY, Wu YL. Prevalence of driver mutations in non-small-cell lung cancers in the People's Republic of China. Lung Cancer (Auckl). 2014;5:1-9.

7. Fiala O, Pesek M, Finek J, Benesova L, Belsanova B, Minarik M. The dominant role of G12C over other KRAS mutation types in the negative prediction of efficacy of epidermal growth factor receptor tyrosine kinase inhibitors in non-small cell lung cancer. Cancer Genet. 2013;206:26-31.

8. Janne PA, Smith I, McWalter G, Mann H, Dougherty B, Walker J, et al. Impact of KRAS codon subtypes from a randomised phase II trial of selumetinib plus docetaxel in KRAS mutant advanced non-small-cell lung cancer. $\mathrm{Br}$ J Cancer. 2015:113:199-203.

9. Janne PA, van den Heuvel MM, Barlesi F, Cobo M, Mazieres J, Crino L, et al. Selumetinib plus Docetaxel compared with Docetaxel alone and progression-free survival in patients with KRAS-mutant advanced non-small cell lung Cancer: the SELECT-1 randomized clinical trial. JAMA. 2017;317: 1844-53.

10. Kaufman J, Stinchcombe TE. Treatment of KRAS-mutant non-small cell lung Cancer: the end of the beginning for targeted therapies. JAMA. 2017;317: 1835-7

11. Skoulidis F, Byers LA, Diao L, Papadimitrakopoulou VA, Tong P, Izzo J, et al. Co-occurring genomic alterations define major subsets of KRAS-mutant lung adenocarcinoma with distinct biology, immune profiles, and therapeutic vulnerabilities. Cancer Discov. 2015;5:860-77. 
12. Dong ZY, Zhong WZ, Zhang XC, Su J, Xie Z, Liu SY, et al. Potential predictive value of TP53 and KRAS mutation status for response to PD-1 blockade immunotherapy in lung adenocarcinoma. Clin Cancer Res. 2017; 23:3012-24.

13. Canon J, Rex K, Saiki AY, Mohr C, Cooke K, Bagal D, et al. The clinical KRAS(G12C) inhibitor AMG 510 drives anti-tumour immunity. Nature. 2019; 575:217-23.

14. Sh IO, Koczywas M, Ulahannan S, Janne PA, Pacheco JM, Burris HA, et al. The SHP2 inhibitor RMC-4630 in patients with KRAS-mutant non-small cell lung Cancer: preliminary evaluation of a first-in-man phase 1 clinical trial. $J$ Thorac Oncol. 2020;15:A12.

15. Hallin J, Engstrom LD, Hargis L, Calinisan A, Aranda R, Briere DM, et al. The KRAS(G12C) inhibitor MRTX849 provides insight toward therapeutic susceptibility of KRAS-mutant cancers in mouse models and patients. Cancer Discov. 2020;10:54-71.

16. Aredo JV, Padda SK, Kunder CA, Han SS, Neal JW, Shrager JB, et al. Impact of KRAS mutation subtype and concurrent pathogenic mutations on non-small cell lung cancer outcomes. Lung Cancer. 2019;133:144-50.

17. El Osta B, Behera M, Kim S, Berry LD, Sica G, Pillai RN, et al. Characteristics and outcomes of patients with metastatic KRAS-mutant lung adenocarcinomas: the lung Cancer mutation consortium experience. J Thorac Oncol. 2019;14:876-89.

18. Scheffler M, Ihle MA, Hein R, Merkelbach-Bruse S, Scheel AH, Siemanowski J, et al. K-ras mutation subtypes in NSCLC and associated co-occuring mutations in other oncogenic pathways. J Thorac Oncol. 2019;14:606-16.

19. Jia $Y$, Jiang $T$, Li X, Zhao C, Zhang L, Zhao $S$, et al. Characterization of distinct types of KRAS mutation and its impact on first-line platinum-based chemotherapy in Chinese patients with advanced non-small cell lung cancer. Oncol Lett. 2017;14:6525-32.

20. Tu HY, Ke EE, Yang JJ, Sun YL, Yan HH, Zheng MY, et al. A comprehensive review of uncommon EGFR mutations in patients with non-small cell lung cancer. Lung Cancer. 2017;114:96-102.

21. Izar B, Zhou H, Heist RS, Azzoli CG, Muzikansky A, Scribner EE, et al. The prognostic impact of KRAS, its codon and amino acid specific mutations, on survival in resected stage I lung adenocarcinoma. J Thorac Oncol. 2014;9: 1363-9.

22. Nadal E, Chen G, Prensner JR, Shiratsuchi H, Sam C, Zhao L, et al. KRASG12C mutation is associated with poor outcome in surgically resected lung adenocarcinoma. J Thorac Oncol. 2014;9:1513-22.

23. Zer A, Ding K, Lee SM, Goss GD, Seymour L, Ellis PM, et al. Pooled analysis of the prognostic and predictive value of KRAS mutation status and mutation subtype in patients with non-small cell lung Cancer treated with epidermal growth factor receptor tyrosine kinase inhibitors. J Thorac Oncol. 2016;11:312-23.

24. Dogan $S$, Shen $R$, Ang DC, Johnson ML, D'Angelo SP, Paik PK, et al. Molecular epidemiology of EGFR and KRAS mutations in 3,026 lung adenocarcinomas: higher susceptibility of women to smoking-related KRASmutant cancers. Clin Cancer Res. 2012;18:6169-77.

25. Guan JL, Zhong WZ, An SJ, Yang JJ, Su J, Chen ZH, et al. KRAS mutation in patients with lung cancer: a predictor for poor prognosis but not for EGFRTKls or chemotherapy. Ann Surg Oncol. 2013;20:1381-8.

26. Svaton M, Fiala O, Pesek M, Bortlicek Z, Minarik M, Benesova L, et al. The prognostic role of KRAS mutation in patients with advanced NSCLC treated with second- or third-line chemotherapy. Anticancer Res. 2016;36:1077-82.

\section{Publisher's Note}

Springer Nature remains neutral with regard to jurisdictional claims in published maps and institutional affiliations.

Ready to submit your research? Choose BMC and benefit from:
- fast, convenient online submission
- thorough peer review by experienced researchers in your field
- rapid publication on acceptance
- support for research data, including large and complex data types
- gold Open Access which fosters wider collaboration and increased citations
- maximum visibility for your research: over 100M website views per year
At BMC, research is always in progress.
Learn more biomedcentral.com/submissions

